\title{
IMAGING SYSTEM PERFORMANCE ANALYSIS USING MODULATION TRANSFER FUNCTION
}

\author{
Nedeljko Pađen ${ }^{1,2, *}$, \\ Dragana Perić2 \\ Milan Milosavljević ${ }^{1}$
}

${ }^{1}$ Singidunum University,

Belgrade, Serbia

2Vlatacom institute,

Belgrade, Serbia
Correspondence:

Nedeljko Pađen

e-mail:

nedeljko.padjen@vlatacom.com

\begin{abstract}
:
Modulation Transfer Function (MTF) is a useful tool for imaging system performance analysis. It can be used as engineering approach for verification of main system parameters, or for optimization task of the system under test. This methodology is based on linear systems theory and allows performance analysis to be divided into subsystems. In this paper, MTF methodology will be presented and explained, as an analytical model and as a testing tool which is implemented in electro-optical laboratory. According to MTF model, calculations are done to provide theoretical limit for the imaging systems. After tests in electro-optical laboratory have been done, we compared model expectations with measurements results and discussed achieved relations. The measurement has shown that the calculated cutoff frequencies correspond to the measured ones, in majority of cases. The calculated MTF curve has proven to be limit for the real measured system performance. Therefore, this study has shown that MTF can be convenient for finding system limitations, bottlenecks and increasing the overall performance of the system. Guidelines for further optimization of the imaging systems are derived.
\end{abstract}

Keywords:

Modulation Transfer Function, infrared, visible, multi-sensor imaging system.

\section{INTRODUCTION}

Today's modern society is facing many challenges in the field of border security, such as the cross-border criminal, smuggling of contraband, illegal immigration, human trafficking, and many others [1]. The protection of these vast areas is usually requiring the integration and centralized control of many type of sensors, giving very different type of data (textual data streams, video, audio, imaging, etc..), which are gathered with different type of devices, such as cameras, radars, motion sensors on smart fences, and similar. One of the key roles in these systems are MultiSensor Imaging Systems (MSIS), which are the sets of different cameras covering visible spectral band - VIS (0.4-0.7 $\mu \mathrm{m})$, but also Near InfraRed - NIR (0.7-1.1 $\mu \mathrm{m})$, Shortwave infrared - SWIR (1.1-2.5 $\mu \mathrm{m})$, Midwave Infrared - MWIR (2.5-7 $\mu \mathrm{m})$ and Longwave Infrared - LWIR $(7-15 \mu \mathrm{m})$. By integrating high quality detectors and powerful lenses, these systems can provide 24 hours visibility, even in the scenarios of degraded 
atmospheric conditions. Based on their main role in the system, MSIS can be designed for various tasks, such as detection, recognition and identification of different type of objects (vehicles, truck, pedestrians, etc.).

This paper will analyze one such multi-sensor imaging system, consisting of visible camera and SWIR camera with lenses, whose specifications will be listed in chapter IV of this paper. This particular system was tested in the electro-optical laboratory, where the performance analysis was done using Modulation Transfer Function (MTF).

In chapter II we will describe the basics of electrooptical (EO) imaging system performance, followed by Chapter III with an overview of the theory behind the MTF analysis and its contribution in the overall performance analysis of one imaging system. In Chapter IV we will describe the electro-optical laboratory, equipment used for the measurements and the procedures and methods used in this process. Chapter V will present the results of the measurements for both visible and SWIR camera, with different focal lengths, and discuss the results in relation to the theoretical expectation. In Chapter VI we will present the result of the outdoor camera performance, where we have choose a scene at distance of $12 \mathrm{~km}$ from the camera position, to demonstrate the system ability to perform detection, recognition and identification of the objects. Finally, in Chapter VII we summarize the conclusions on the conducted testing and propose some possible guidelines for further optimization of the electro-optical imaging system.

\section{EO IMAGING SYSTEM PERFORMANCE}

Performance analysis of an electro-optical imaging system is a complex process where the real system optimization can be achieved only if an end-to-end analysis of the whole system is done, from the scene, to the observer [2]. Figure 1 illustrates one such system, by presenting standard elements involved in the final creation of the image of the object, to be presented to the observer.

The resulting image quality is affected by various effects, such as [2]:

- The scene content - background, target and background characteristics, motion, clutter,

- Intervening atmosphere - transmittance, rain, haze/fog, dust,

- Electro-optical system - resolution, sensitivity, noise, Minimum Resolvable Temperature (for infrared systems), Minimum resolvable Contrast (for visible systems),
- Display - luminance, contrast, distance for observer, glare and finally,

- Observer - his experience, training, fatigue, workload.

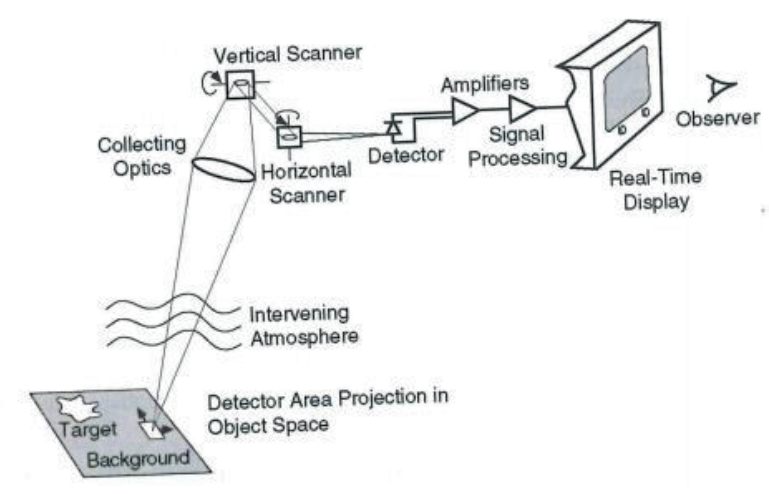

Figure 1. Electro-optical system [2]

With all these variables, different models are developed for the electro-optical systems, with the task to relate measurable system design parameters, with their operational performance. Three levels of models are fulfilling these requirements [3], [4]:

- Component/phenomenology models - These models are based on finding the MTF of the individual elements, which will give us the MTF of the whole system $\left(\mathrm{MTF}_{\mathrm{SYS}}\right)$. MTF $\mathrm{MYS}_{\text {SY }}$ will then be used as the input for the next-level system modeling

- System performance models - Built on component models, they describe the total system performance for some controlled tasks

- Operational models - These models characterize the overall operational system functionality, where they are used to calculate detection, recognition and identification ranges

The focus of our analysis will be restricted to the component model, where the Modulation Transfer Function will help us describe the signal transfer characteristics of our multi-sensor imaging system. The MTF will be described in more detail in the following chapter.

\section{MTF ANALYSIS}

The creation of an image can be mathematically described as a convolution operation (denoted by ${ }^{*}$ ) of an ideal image $\mathrm{f}(\mathrm{x}, \mathrm{y})$, convolved with the total impulse response of the electro-optical systems $\mathrm{h}(\mathrm{x}, \mathrm{y})$. 
$g(x, y)=f(x, y) * h(x, y)$

where the total impulse response is the summation of weighted impulse responses of different elements [5]. Thus, the systems can be analyzed directly in the spatial (time) domain, which is involving a complex mathematics. The alternative to this is the analysis in the frequency-domain, by means of Fourier analysis, where the irradiance distribution in the object or image plane of a time-domain electrical signal is transferred to a "spatial frequencies" in the frequency domain. In that way, we will have:

$G(x, y)=F(x, y) x H(x, y)$

representing the Fourier transform of the elements of the equation (1). This movement from time to spectral domain is allowing much effective analysis of spectral response of the system, where the $\mathrm{H}(\mathrm{x}, \mathrm{y})$ is referred as the Optical Transfer Function (OTF) [5].

The Modulation Transfer Function represents the modulo of OTF, i.e. the magnitude response of our optical system to the sinusoidal input signals of various frequencies.

This kind of analysis can be performed only for linear, shift-invariant systems [2]. Although these conditions are not always fulfilled, the MTF analysis is very useful tool in a system performance analysis and comparison and, as such, very much in use in the system design and choice of the adequate optical elements [6].

Modulation in optical system terminology refers to the contrast between the bright and dark regions of an image - high spatial frequencies relates to good contrast, low spatial frequency are referring to degraded contrast [5]. The MTF are basically plotting the modulation (contrast) versus spatial frequency. The following figure depicts the degradation of the MTF function as the result of increased spatial frequency:

By moving to the spectral domain, instead of convoluting the independent impulse responses of the system components, we will simply multiply their separately calculated MTFs, resulting in the overall system modulation transfer function $\left(\mathrm{MTF}_{\mathrm{SYS}}\right)$. The Figure 3 is illustrating typical MTF shapes of some components.

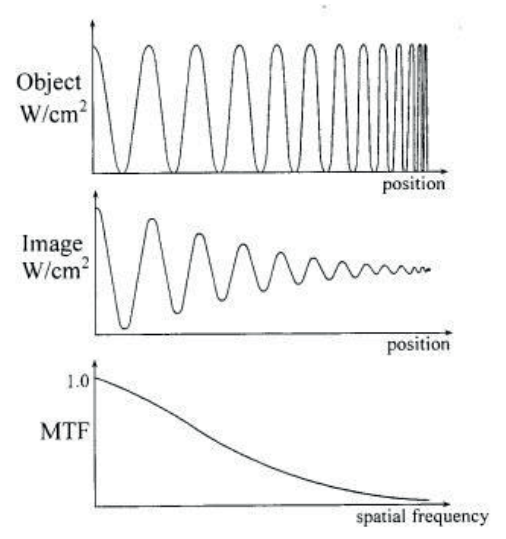

Figure 2. MTF as a function of spatial frequency [5]

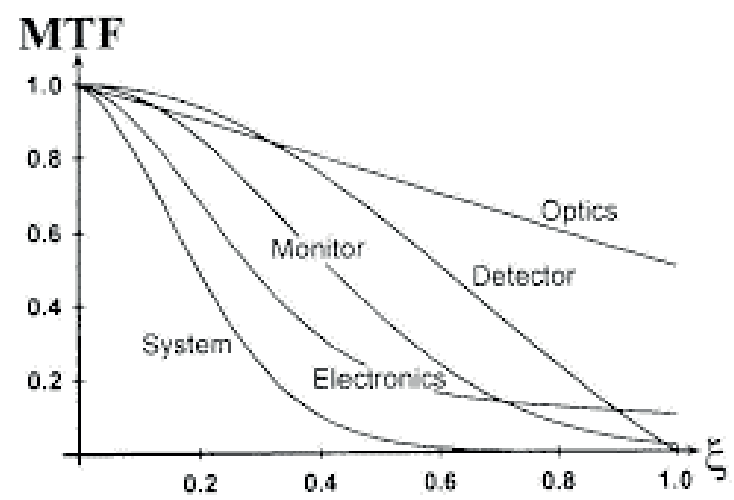

Figure 3. System MTF as a result of components MTFs [5]

The MTFs presented on figure 3 do not conclude the list of the elements affecting the final shape of the MTF system graph, where the jitter, defocus and noise are also influencing the final result. The more components are taken under the analysis, the better result (result closer to real measurements) will be achieved. As a rule of the thumb, it can be considered that the quality of the optical system is better, if the area below the curve is greater. None the less, there is no ultimate way to evaluate which MTF shape is the best [5], due to non-linearity of the human eye which does the task of reconstruction filtering. For that reason, the results from the laboratory should be accompanied with the tests performed in the real environment, which should enhance the evaluation procedure. 
Our analysis will focus on the MTF for detector (determined by the size of the pixel and the focal length of the lens) and MTF for the optical system (which is limited by the optical diffraction). The description of the laboratory and the methodology used in this process will be described in the following chapter.

\section{MTF MEASUREMENT SETUP}

The measurements of the MTF characteristics were done in an electro-optical laboratory equipped with collimator station, illustrated on the following figure:

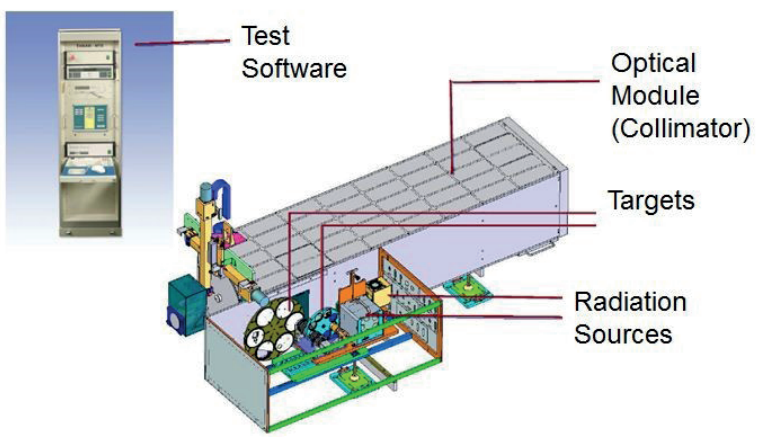

Figure 4. Electro-optical modular test station [7]

The test system has the following characteristics [7]: Collimator's effective focal length (EFL) is 120 inch, with the clear aperture of $14 \mathrm{inch}$. The field of view is $1.0^{\circ}$ and the radiation source is VIS/SWIR integration sphere $0.44-2.2 \mu \mathrm{m}$.

The characteristics of the cameras under the test are as follows:

- SWIR - resolution 640 x512 pixels, detector pixel size $15 \mu \mathrm{m}$. Lens has declared waveband 0.9-1.7 $\mu \mathrm{m}$, and variable focal length $\left(f_{1}\right)$ of up to $1500 \mathrm{~mm}$. The measurements were made on $\mathrm{f}_{1}=$ $500 \mathrm{~mm}$, with F\#4, and FL $=2500 \mathrm{~mm}$, with F\#16

- Visible camera - resolution 1920x1080 pixels, detector pixel size $5 \mu \mathrm{m}$. Lens has declared waveband $0.4-0.7 \mu \mathrm{m}$, and the variable focal length of up to $2000 \mathrm{~mm}$ (with extender). The measurements were made on $\mathrm{f}_{1}=1000 \mathrm{~mm}$, with F\#7.2, and $\mathrm{f}_{1}=2000 \mathrm{~mm}$, with $\mathrm{F} \# 16$

The target used for the MTF measurement is steptarget, depicted in the figure 5 . The MTF measurement procedure with step target is described in [8].

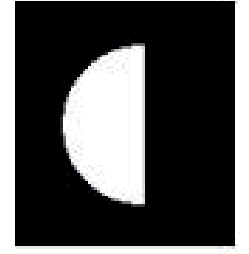

Figure 5. Step-target, used in MTF measurement

While the target has almost perfect edge, its image gets distorted as a result of the system imperfection, resulting in the line spread function (LSF).

The measurement process begins by placing (and selecting) the step target in the target wheel and switching on the integration sphere and setting the intensity. Images in the number of consecutive frames are taken, and averaging is done over all recorded frames. After that the edges are aligned, to do the averaging over the lines from where the above mentioned LSF is calculated. From LSF, by means of Fast Fourier transformation (FFT) the MTF graph is derived, presenting all the frequencies, up to cut-off frequency.

The MTF curve for the detector is the magnitude of the following formula

$$
\begin{aligned}
& \operatorname{OTF}_{\text {detector }}\left(f_{x}\right)=\operatorname{sinc}\left(\alpha f_{x}\right)=\frac{\sin \left(\pi \alpha f_{x}\right)}{\pi \alpha f_{x}} \text {, where } \\
& \alpha=\frac{1}{f d c o}=\frac{d}{f_{l}}
\end{aligned}
$$

Diffraction (optical) MTF was calculated by the following formula:

$$
\begin{aligned}
& \text { OTF } F_{\text {diff }}=\frac{2}{\pi}\left[\cos ^{-1}\left(\frac{f_{x}}{f_{\text {oco }}}\right)-\left(\frac{f_{x}}{f_{\text {oco }}}\right) \sqrt{1-\left(\frac{f_{x}}{f_{\text {oco }}}\right)^{2}} \text {, for } f_{x} \leq f_{\text {oco }}\right. \\
& \text { OTF } F_{\text {diff }}=0, \text { for } f_{x}>f_{\text {oco }} \quad, \text { where } \\
& f_{\text {oco }}=\frac{1}{\lambda F_{\#}} \text { and } F_{\#}=\frac{f_{l}}{D}
\end{aligned}
$$

The parameters used in the formulas (and in the tables in the following chapter) are

- $\boldsymbol{\lambda}$ - central wavelength

- $\mathbf{d}$ - detector pixel size

- $\mathbf{f}_{1}$ - focal length

- D - Diameter of the lens aperture

- F\# - function of focal length and lens aperture 
- f_dco - detector cutoff frequency

- f_oco - optical (diffraction) cutoff frequency, due to lens diffraction [1]

Prior to the measurement process the MTF calibration was performed, with the analysis of dominating limitation effects for the electro-optical system. These limitations are pixel size and lens focal length, for the detector-limited systems and lens numerical aperture (NA) and working imager spectral band, for diffraction limited systems.

The results of the conducted measurements, with the discussion of the results in relation to the theoretical calculation are given in the following chapter.

\section{MEASUREMENT RESULTS}

The measurement parameters and the calculated cutoff frequencies are summarized in Table I, for visible camera, and Table II, for short-wave infrared camera.

Table 1. Calculated cut-off frequencies for visible camera

\begin{tabular}{|c|c|c|c|c|c|}
\hline$\lambda[\mathrm{m}]$ & d_det [jm] & fl [mm] & F\# & $\begin{array}{c}\text { f_dco } \\
{[\mathrm{cy} / \mathrm{mRad}]}\end{array}$ & $\begin{array}{c}f_{-} \text {oco } \\
{[\mathrm{cy} / \mathrm{mRad}]}\end{array}$ \\
\hline D.5B & 5.6B & 1, BEBA.EB & 7.28 & 100.00 & 277.78 \\
\hline B.5B & 5.6B & 2,EBE.GB & 16.68 & 200.00 & 125.00 \\
\hline
\end{tabular}

Table 2. Calculated cut-off frequencies for swir camera

\begin{tabular}{|c|c|c|c|c|c|}
\hline$\lambda[u m]$ & d_det [jm] & fl [mm] & F\# & $\begin{array}{c}\text { f_dco } \\
\text { [cy/mRad] }\end{array}$ & $\begin{array}{c}\text { f_oco } \\
\text { [cy/mRad] }\end{array}$ \\
\hline 1.50 & 15.60 & 5ER.6B & 4.60 & 16.67 & 166.67 \\
\hline 1.50 & 15.68 & 2,5EB.BB & 16.6B & 83.33 & 41.67 \\
\hline
\end{tabular}

These values were then used to calculate and plot the graphs for the MTF of detector, MTF of diffraction, and the resulted MTF of the system (MTF product) using the formulas (3-7) given in previous chapter.

Figure 6 is presenting the graphs of the calculated MTFs and the measured MTF for visible camera without extender $\left(\mathrm{f}_{1}=1000 \mathrm{~mm}\right)$, while the figure 7 is depicting the results for visible camera with extender $\left(f_{1}\right.$ $=2000 \mathrm{~mm}$ ).

Figure 8 is presenting the graphs of the calculated MTFs and the measured MTF for SWIR camera with focal length of $500 \mathrm{~mm}$, and finally figure 9 is giving the graphs of SWIR with $\mathrm{f}_{1}=2500 \mathrm{~mm}$.

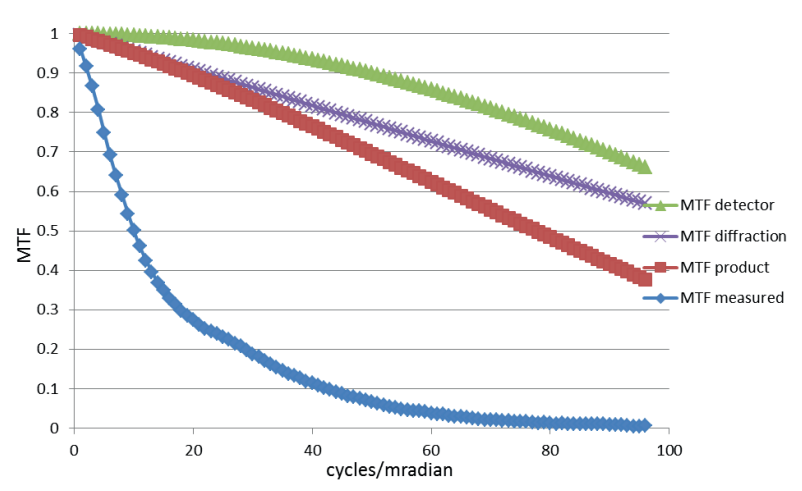

Figure 6. MTF for visible camera, $\mathrm{f}_{1}=1000 \mathrm{~mm}$

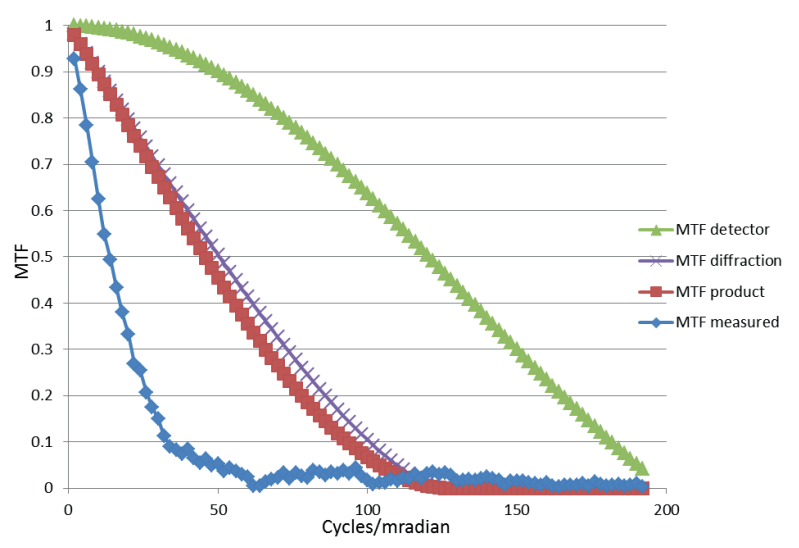

Figure 7. MTF for visible camera, $\mathrm{f}_{1}=2000 \mathrm{~mm}$

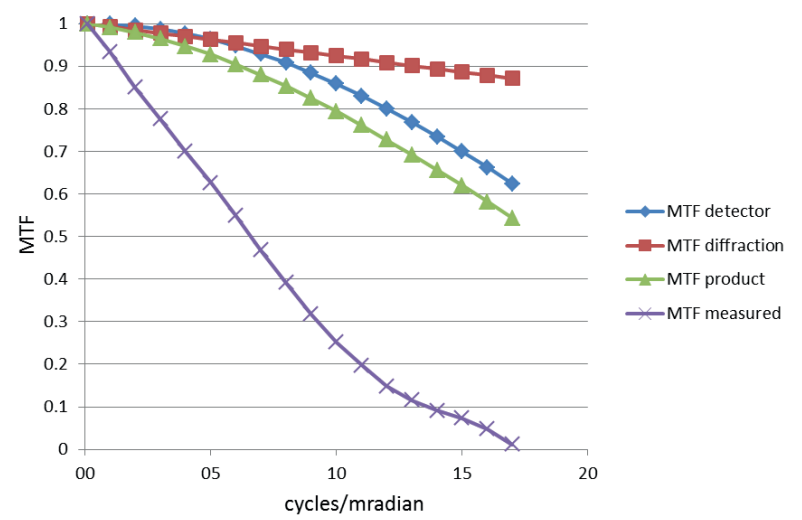

Figure 8. MTF for SWIR camera, $\mathrm{f}_{1}=500 \mathrm{~mm}$ 


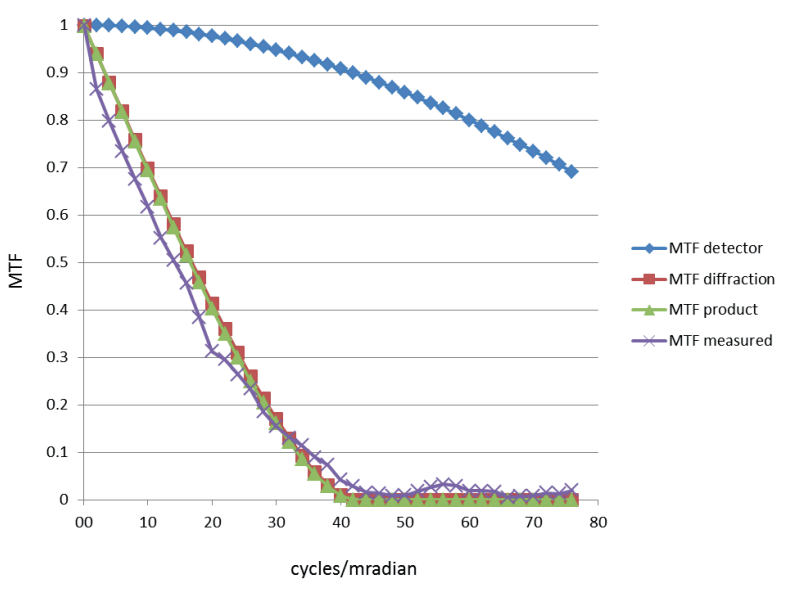

Figure 9. MTF for SWIR camera, $\mathrm{f}_{1}=2500 \mathrm{~mm}$

What follows is the discussion of these results.

\section{A. Visible camera, without extender $(\mathrm{fl}=1000 \mathrm{~mm})$}

By analyzing results presented in Figure 6, we can conclude that the measured Modular Transfer Function has the expected cut-off frequency, which is in this case limited by the detector pixel size. The deviation of the measured MTF from the theoretical one, reflected in the steeper decline of the MTF curve, can be explained by the effect of the elements which were not measured in this case, such as focus, electronics for video processing, display, etc. We can notice that MTF drops below $50 \%$ at approximately tenth of the cut-off frequency.

\section{B. Visible camera, with extender $(f l=2000 \mathrm{~mm})$}

In the case of visible camera with the extender, depicted in Figure 7, we can see that the measured result (MTF measured curve) has the lower cut-off frequency than the one expected by the theory (MTF product curve). We can also conclude that limiting factor in this case is the diffraction of the lens system. Calculated cutoff frequency as a result of the lens diffraction is 125 cycles/mrad, while the values of the measured MTF are falling below 0.02 already for the spatial frequencies around 60 cycles $/ \mathrm{mrad}$. As in this scenario we have use the optical extender (to achieve the targeted focal length), this deviation can be explained by the aberrations (imperfection) of the optical extender elements.

\section{SWIR camera, focal length $500 \mathrm{~mm}$}

In this case the measured MTF has the expected cutoff frequency, which is limited by the detector pixel size. The same as for the visible camera without extender (measurement $A$ ), the deviation of the measured MTF from the theoretical one, reflected in the steeper decline of the
MTF curve, can be explained by the effect of the elements which were not measured, such as focus, electronics for video processing, display, etc. However, in this case we can notice that MTF drops below 50\% at approximately half of the cut-off frequency. Comparing this result with the one discussed in section A, we conclude that SWIR lens has better optical characteristics that the one used with visible camera. Having this in mind, we expect better identification in SWIR images, which will be tested with images taken from real scenario.

\section{SWIR camera, focal length $2500 \mathrm{~mm}$}

This final measurement, for the short-wave infrared camera with the narrow field of view (NFOV), gave us the best results in terms of matching of the shape of the measured and theoretical MTF curve, which are in this case almost identical. The limitation in this case is optical diffraction of the lens, the MTF curve reaches zero for the spatial frequencies around 40 cycles $/ \mathrm{mrad}$, while the diffraction cut-off frequency is round $41 \mathrm{cy}$ cles $/ \mathrm{mrad}$.

\section{REAL SCENARIO IMAGES}

In order to illustrate image performance of the multi-sensor imaging system (MSIS) in real scenario, the system tested in the laboratory was installed outdoor and set to monitor the scene approximately 12 kilometers apart from the MSIS position.

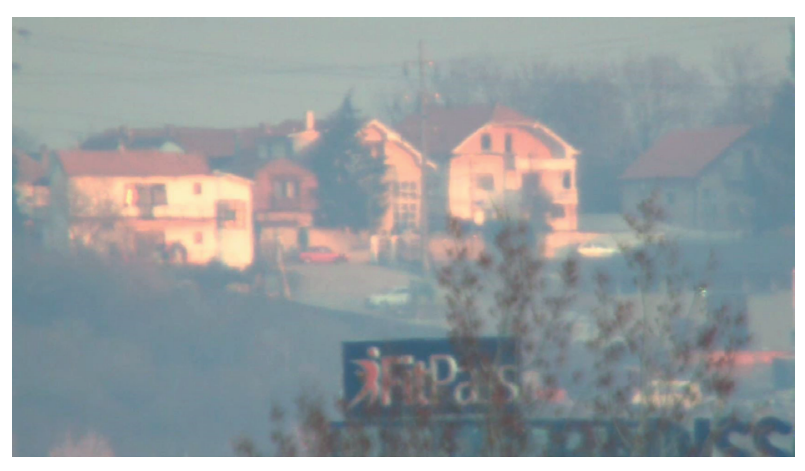

Figure 10. Visible camera image

What is clearly distinguishable on the pictures are the vehicles, buses, pedestrians and the general background characteristics (buildings, trees, etc..). Since this type of system is generally designed to perform detection, recognition and identification of the objects, it is safe to say that the system is performing well, for the 
purpose it was built for. Comparing images in VIS and SWIR, we can conclude that SWIR image is richer in details, which is expectable regarding the comment stated in section C. During the tests we also noticed remarkable advantage of the SWIR image in presence of fog.

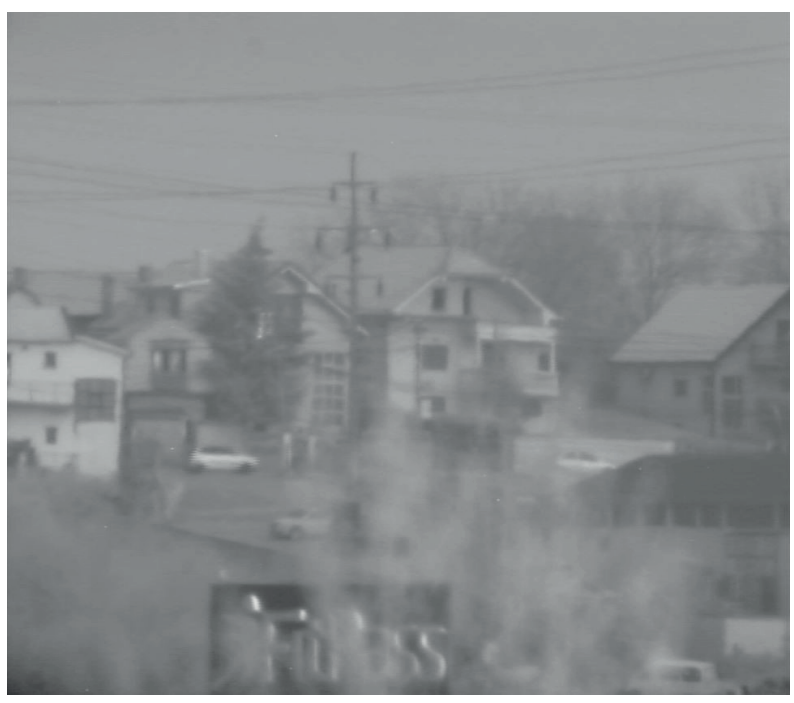

Figure 11. SWIR camera image

\section{CONCLUSIONS}

The theoretical analysis and laboratory measurements of electro-optical system performance have demonstrated that MTF can be an effective analytical tool.

Theoretical calculations have shown that the increase of focal length results in the increase of f-number $(\mathrm{F} \#)$, and for that reason the diffraction of the lens system becomes the dominant limitation factor (7), compare to the detector limitation. In that way, we have identified the maximal frequency for our system. On the other side, when the focal length is small, the system limitation is imposed by the detector pixel size, which indicates how the system can be further improved.

MTF measurements in the electro-optical laboratory have also given some valuable information. They have confirmed that by increasing the focal length, the system is moving from detector-limited, to diffraction limited system. For lower frequencies, the steeper curve of the measured MTF can be explained by the effects of the elements not measured in this case. The comparison of these curves for VIS and SWIR camera has shown that SWIR lens has better optical characteristics, which is another valuable result of the MTF analysis.
With the real scenario images we have confirmed the expectation from the laboratory measurements that SWIR camera gives better (richer in detail) image. This was especially obvious for the tests conducted in the degraded environmental conditions (fog).

Taking into account the considerable distance, it can also be concluded that the whole EO system is performing well, for the purpose it was built for (detection, recognition and identification of the objects). This leads to the general guideline in the design and optimization of EO systems - the key of the success is to fully understand the system requirements and the use-cases [9], since no system can be designed to provide perfect resolution, contrast, brightness and color fidelity, for any object distance, in any environmental condition. Therefore, the best systems are the ones designed for the exact purpose. Then, through the careful selection of system elements (lens, detector, etc..) and the system parameter optimization we can influence on the system performance.

To further prove the value of MTF analysis, future efforts will be made to include in the calculations the effects of the other system elements, such as focus, jitter and image processing MTFs. It is expected to provide better matching of measured and calculated MTF results, but also to give some additional direction for further EO system optimization.

\section{REFERENCES}

[1] McDaniel Robert, New sensors enhance border security, SPIE Newsroom. DOI: 10.1117/2.1200609.0300, 2006

[2] G. Holst, Electro-optical imaging system performance, $5^{\text {th }}$ ed, JCD Publishing, Winter Park, FL, 2008, chapter 1.

[3] D. L. Shumaker, J. T. Wood, Overwiev of current IR analysis capabilities and problem areas, Infrared Systems and Components II, H. M. Liaw, ed., SPIE Proceedings Vol. 890, pp.74-80 (1988)

[4] Robert D. Fiete, Modeling the imaging chain of digital cameras, SPIE Press, tutorial text, Volume TT92, ISBN 978-0-8194-8339-3, 2010

[5] G. Boreman, Modulation transfer function in optical and electrooptical systems, SPIE, Tutorial texts in optical engineering, v. TT 52, 2001.

[6] D. Peric, B. Livada, M. Radisavljevic, S. Vujic, "Performance Comparison of SWIR Vision Systems Using MTF Analysis", Proceedings of $5^{\text {th }}$ International Conference of Electrical, Electronics and Computing Engineering, IcETRAN 2018, Palic, Serbia, June 11-14, pp. EKI1.1 
[7] Modular Electro-Optical Test System, CI Systems, Cat. No. 607-5610H, 2012.

[8] G. Holst, Testing and Evaluation of Infrared Imaging Systems, $3^{\text {rd }}$ ed, JCD Publishing, Winter Park, FL, 2008
[9] Philip C. D. Hobbs, Building Electro-Optical Systems: Making it All Work, John Wiley \& Sons, Inc. ISBNs: 0-471-24681-6 (Hardback); 0-471-22429-4 (Electronic), p.359 